Europhysics Letters

PREPRINT

\title{
Fluctuations of a driven membrane in an electrolyte
}

\author{
D. Lacoste $^{1}$, M. Cosentino Lagomarsino ${ }^{2,3}$, And JF. Joanny ${ }^{2}$ \\ 1 Laboratoire Physico-Chimie Théorique, ESPCI, 10 rue Vauquelin 75005 Paris, France \\ 2 UMR 168 / Institut Curie, 26 rue d'Ulm 75005 Paris, France \\ 3 Università degli Studi di Milano, Dip. Fisica, Via Celoria 16, 20133 Milano, Italy
}

PACS. 87.16.b - Subcellular structure and processes..

PACS. 05.40.a - Fluctuation phenomena, random processes, noise and Brownian motion..

PACS. 05.70.Np - Interface and surface thermodynamics.

\begin{abstract}
We develop a model for a driven cell- or artificial membrane in an electrolyte. The system is kept far from equilibrium by the application of a DC electric field or by concentration gradients, which causes ions to flow through specific ion-conducting units (representing pumps, channels or natural pores). We consider the case of planar geometry and Debye-Hückel regime, and obtain the membrane equation of motion within Stokes hydrodynamics. At steady state, the applied field causes an accumulation of charges close to the membrane, which, similarly to the equilibrium case, can be described with renormalized membrane tension and bending modulus. However, as opposed to the equilibrium situation, we find new terms in the membrane equation of motion, which arise specifically in the out-of-equilibrium case. We show that these terms lead in certain conditions to instabilities.
\end{abstract}

Introduction. - Phospholipid membranes are a major constituent of the cell. They cover the surface of all organelles and play an important role in many fundamental cellular processes such as intracellular transport [1]. At a mesoscopic scale, the equilibrium properties of fluid membranes can be accounted for by the so-called Helfrich Hamiltonian, which describes the mechanics of the membrane in terms of parameters, such as membrane tension, bending modulus and spontaneous curvature. These mechanical parameters strongly depend on the electrostatic charge of the phospholipid molecules. The electrostatic contributions to the bending modulus and surface tension for a fluid membrane have been obtained by several authors [2-4] using free energy calculations for simple geometries (sphere, cylinders, planes).

Real membranes are non-equilibrium systems. They are in general active in the sense that they are constantly maintained out of equilibrium either by active proteins (such as ATP-consuming enzymes) inside the membrane or by an energy flow due to external parameters (such as a lipid flux [5]). An example of model active membrane has been proposed in Ref. [6]. In these experiments, a giant unilamellar vesicle is activated by the inclusion of bacteriorhodopsin pumps, which transfer protons unidirectionally across the membrane by undergoing light-activated conformational changes. In the same work, a hydrodynamic theory has also been developed to calculate the nonequilibrium fluctuations of the membrane, induced by the activity of the pumps. In this paper, we study theoretically an electrically neutral membrane containing passive ion channels in an electrolyte solution and driven out

(c) EDP Sciences 

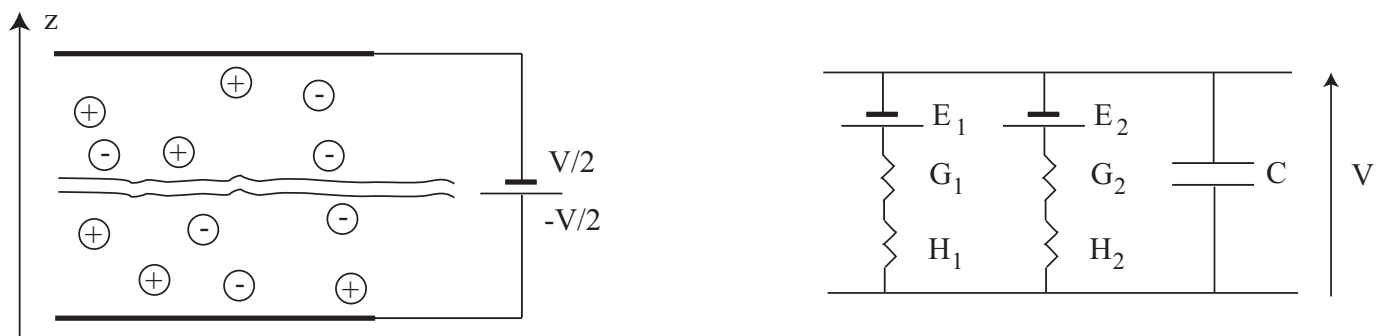

Fig. 1 - Left: Sketch of a quasi-planar fluid membrane embedded in an electrolyte in the presence of an applied potential difference and concentration gradients (not represented). Right: equivalent electrical circuit, with the contribution of the two ions $k=1,2$ in parallel, and for each ion, two conductances $\left(G_{k}\right.$ for the membrane and $H_{k}$ for the electrolyte) and an electromotive force $E_{k}$ in series. When the finite thickness of the bilayer is included, there is also a capacitor $C$ in parallel which contains contributions from Debye layers and from the membrane.

of equilibrium by the application of a DC electric field or by ion concentration gradients [7] (Fig. (1). As in refs. [6,8,9], we use a generalized hydrodynamic description appropriate for low Reynolds number, that we couple to the electrokinetic equations [10] within Debye-Hückel approximation. We calculate the undulation fluctuations by expansion around the planar state of the membrane. With minor modifications, our method can also be used to calculate the electrical contribution to the fluctuations of a membrane containing pumps. This contribution, was not taken into account in ref. [6]. We first consider the case of a membrane of zero thickness, and then generalize the model to the case of a bilayer with a finite thickness and a finite dielectric constant, lower than that of the solvent. The later model is more realistic since it contains both capacitive effects and ions transport $[11,12]$.

Membrane of zero thickness driven by the application of an electrostatic potential difference. - In this section, we compute the electrostatic potential $\phi$, and then use the electrostatic forces as a source term in the hydrodynamic equations to obtain the membrane equation of motion. We use a linear theory and assume a steady state. The quasi-planar membrane is located in the plane $z=0$ (Fig. (1), it is embedded in an electrolyte and carries passive channels for two types of monovalent ions. There is an imposed potential difference $V$ across the system of length $L$. The boundary conditions for the potential are therefore $\phi(z= \pm L / 2)= \pm V / 2$ and $\partial_{z} \phi\left(z \rightarrow 0^{ \pm}\right)=0$ due to the assumption of a vanishing membrane dielectric constant. We denote $c_{k}$ the concentrations of the two ions, where the index $k$ is 1 for the positive ion $\left(z_{1}=1\right)$ and is 2 for the negative ion $\left(z_{2}=-1\right)$. Far from the membrane these concentrations are fixed, so that $c_{k}^{ \pm}(z= \pm L / 2)=n^{ \pm}$, where the superscript + (resp. - ) denotes $z>0$ (resp. $z<0$ ). Everywhere in the electrolyte we linearize the concentrations around these bulk values. A point on the membrane is characterized by a $2 \mathrm{D}$ vector field $\mathbf{r}_{\perp}$ and for small undulations a height function $h\left(\mathbf{r}_{\perp}\right)$. The inverse Debye-Hückel length is $\kappa^{+}$on the positive side (resp. $\kappa^{-}$on the negative side) with $\kappa^{ \pm 2}=2 e^{2} n^{ \pm} / \epsilon k_{B} T$. Within a linear theory for the concentrations and the potential, the fluxes of the positive ions of bulk diffusion coefficient $D_{1}$ and of the negative ions of diffusion coefficient $D_{2}$ are proportional to the ion chemical potential gradients

$$
\begin{aligned}
& \mathbf{J}_{1}^{ \pm}=-n^{ \pm} D_{1}\left[\nabla \rho_{1}^{ \pm}+\nabla \psi^{ \pm}\right] \\
& \mathbf{J}_{2}^{ \pm}=-n^{ \pm} D_{2}\left[\nabla \rho_{2}^{ \pm}-\nabla \psi^{ \pm}\right],
\end{aligned}
$$


where $\rho_{k}$ are normalized concentrations such that $c_{k}^{ \pm}=n^{ \pm}\left(1+\rho_{k}^{ \pm}\right)$, and $\psi=e \phi / k_{B} T$ is the normalized potential. The charge density is $\rho^{ \pm}=e n^{ \pm}\left(\rho_{1}^{ \pm}-\rho_{2}^{ \pm}\right)$; it obeys Poisson's equation. The conservation of both types of ions in an incompressible fluid imposes that $\partial c_{k} / \partial t=-\nabla \cdot \mathbf{J}_{k}$, and therefore at steady state the ions fluxes are constant. At the membrane surface, the normal bulk flux of each ion species is equal to the flux through the membrane. Throughout this paper, we assume that the membrane has a constant conductance per unit area $G_{k}$ for the two types of ions. In general, the conductance depends in a non linear way on the electrostatic potential difference across the membrane $[11,12]$, but here as a first step we assume linear conductivity. In the case of symmetric concentrations $n^{+}=n^{-}$, the charge density is an odd function of $z$ and the electric field an even function of $z$, but no such symmetry is present in the general case when $n^{+} \neq n^{-}$.

The electrostatic potential and the charge density on the positive (resp. negative) side depends on the surface charge on the positive electrode (on the same side) $\sigma^{+}$(resp. $\sigma^{-}$), which is defined as $\sigma^{ \pm}= \pm \epsilon \partial_{z} \phi(z= \pm L / 2)$. We find that

$$
\begin{aligned}
\sigma^{+} & =-\frac{1}{\kappa^{+2}}\left(\frac{i_{1}}{D_{1}}+\frac{i_{2}}{D_{2}}\right), \\
\sigma^{-} & =\frac{1}{\kappa^{-2}}\left(\frac{i_{1}}{D_{1}}+\frac{i_{2}}{D_{2}}\right),
\end{aligned}
$$

where $i_{1}=e \mathbf{J}_{1} \cdot \hat{\mathbf{e}}_{\mathbf{z}}$ and $i_{2}=-e \mathbf{J}_{2} \cdot \hat{\mathbf{e}}_{\mathbf{z}}$ are the electric currents carried by ion species 1 and 2 . Note that the electroneutrality is satisfied, since $\int_{0}^{\infty} \rho^{+}(z) d z=-\sigma^{+}$and $\int_{-\infty}^{0} \rho^{-}(z) d z=-\sigma^{-}$. The non-vanishing charges of the electrodes compensate the overall charge of the electrolyte. The electric currents (for $L \kappa^{ \pm} \gg 1$ ) are given by

$$
i_{k}=\frac{-G_{k} v_{k}}{1+G_{k} L k_{B} T / D_{k} n e^{2}},
$$

where $v_{k}=V-E_{k}, E_{k}=-k_{B} T \log \left(n^{+} / n^{-}\right) / e z_{k}$ is the Nernst potential of ion $k=1,2$ and $n=2 n^{+} n^{-} /\left(n^{+}+n^{-}\right)$. This equation is consistent with the usual electrical representation of ion channels, shown in fig. 1 1 with the contribution of the two ions in parallel, and for each ion, two conductances $G_{k}$ and $H_{k}$ and an electromotive force $E_{k}$ in series [11,12]. $H_{k}$ corresponds to the bulk conductance per unit area of each ion $H_{k}=D_{k} n e^{2} / k_{B} T L$, which can be itself decomposed in two conductances in series for each side of the electrolyte.

We now consider fluctuations to first order in the membrane undulation $h$, and in a quasisteady state, assuming that the pulsation $\omega<<D_{k} \kappa^{2}$. The calculations are conveniently carried out using Fourier transforms, defined as $f(\mathbf{q}, z)=\int_{-\infty}^{\infty} d^{2} \mathbf{r}_{\perp} e^{i \mathbf{q} \cdot \mathbf{r}_{\perp}} f\left(\mathbf{r}_{\perp}, z\right)$, where $\mathbf{q}$ is the transverse wave vector for membrane fluctuations. Rotational symmetry imposes that all the fields in our problem depend only on $q=|\mathbf{q}|$. We find that, the first order correction to the fluxes given by Eq. 1 vanishes, which indicates that there is no correction to the ion conductivities due to membrane fluctuation at this order. More precisely, the first-order steady-state solution of our electrokinetic equations is then $\phi^{(1)}(q, z)= \pm \kappa^{ \pm} \sigma^{ \pm} h(q) \exp \left(-\kappa_{q}^{ \pm} z\right) / \epsilon \kappa_{q}^{ \pm}$(see Fig. (2) and $\rho^{(1)}(q, z)=\mp \kappa^{ \pm 3} h(q) \exp \left(-\kappa_{q}^{ \pm} z\right) \sigma^{ \pm} / \kappa_{q}^{ \pm}$, where $\kappa_{q}^{ \pm}=\left(q^{2}+\kappa^{ \pm 2}\right)^{1 / 2}$. Having determined perturbatively the ion concentrations and the electric field, we study the membrane undulations imposed by the hydrodynamics of the surrounding electrolyte. The velocity field is obtained from the Stokes equation in the presence of a force density $\mathbf{f}$ acting on the fluid [13],

$$
\eta \nabla^{2} \mathbf{v}-\nabla P+\mathbf{f}=0
$$

with the incompressibility condition $\nabla \cdot \mathbf{v}=0$. Away from the membrane, the boundary condition is $\mathbf{v}\left(\mathbf{r}_{\perp}, z \rightarrow \infty\right)=0$. We assume an absence of permeation through the membrane 

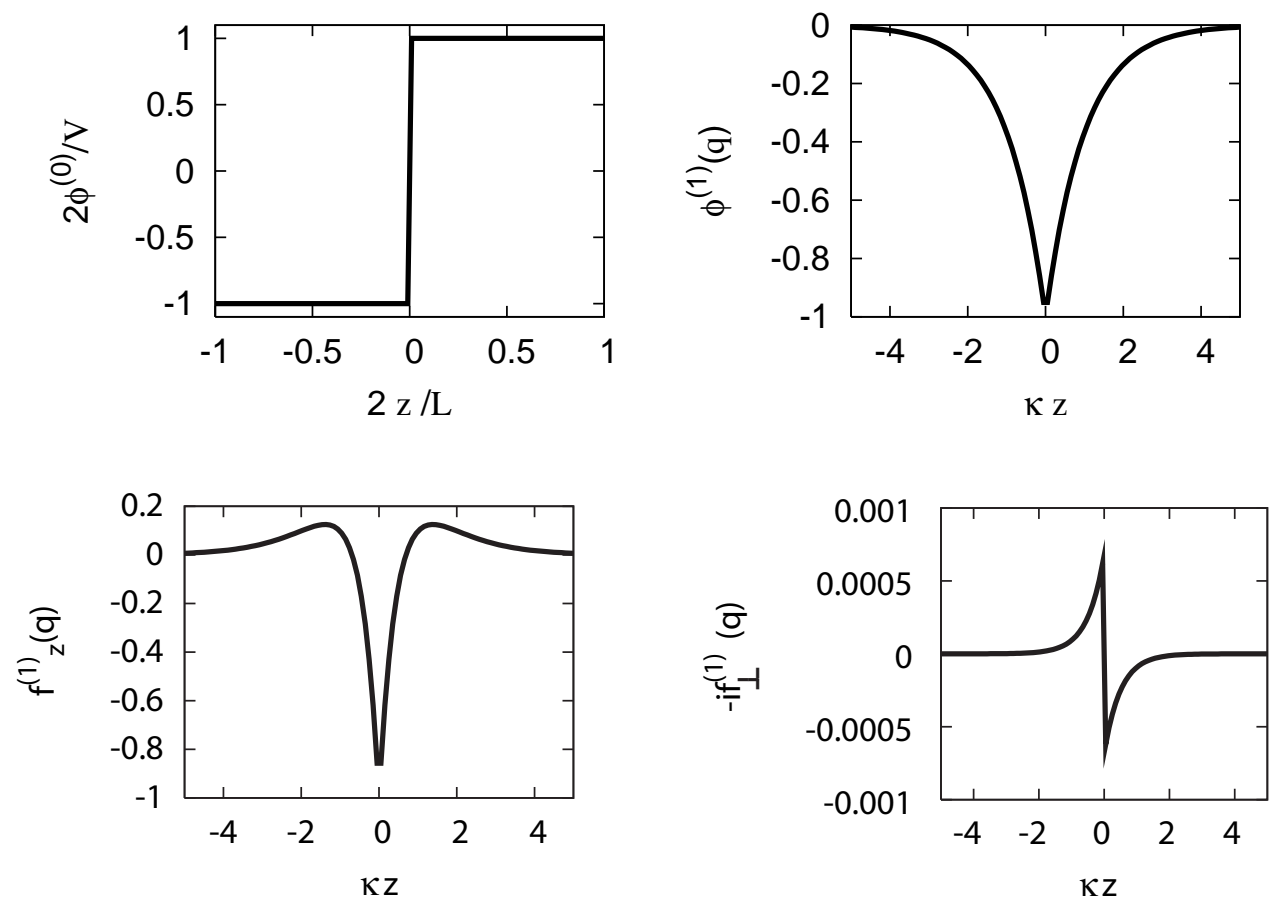

Fig. 2 - Solutions of the electrokinetic equations for a membrane of zero thickness and symmetric ions concentrations $n^{-}=n^{+}=n$. The parameters used in this figure are $V=50 \mathrm{mV}, L=1 \mu \mathrm{m}$, $G_{1}=G_{2}=10 \Omega^{-1} / \mathrm{m}^{2}, D_{1}=D_{2}=10^{-5} \mathrm{~cm}^{2} / \mathrm{s}$ and $n=16.6 \mathrm{mM}$. Top: Left: Normalized potential at zeroth order $2 \phi^{(0)}(z) / V$ as function of $2 z / L$. Right: Normalized potential at first order in membrane undulation $\phi^{(1)}(q, z)$ for a wavevector $q=10^{6} \mathrm{~m}^{-1}$, the normalization factor is $h(q) \sigma 4 \pi / \epsilon$. Bottom: Left: Normalized force along $z$ at first order $f_{z}^{(1)}(q, z)$. Right: Normalized force along $\mathbf{q}$ at first order $-i f_{\perp}^{(1)}(q, z)$ (where $i$ is the imaginary number). This is for the same wavevector and the normalization factor for the forces is $h(q) \sigma^{2} \kappa^{2} 4 \pi / \epsilon$.

and the no-slip condition $\mathbf{v}\left(\mathbf{r}_{\perp}, z=0\right)=\partial_{t} h\left(\mathbf{r}_{\perp}, t\right) \hat{\mathbf{e}}_{\mathbf{z}}$. Here, the active forces [8,9] only act on the ions that are transported across the membrane, i.e. $\mathbf{f}\left(\mathbf{r}_{\perp}, z\right)=\rho\left(\mathbf{r}_{\perp}, z\right) \mathbf{E}\left(\mathbf{r}_{\perp}, z\right)$. The zeroth order contribution to this force $\mathbf{f}^{(0)}$ is balanced by the pressure gradient of Eq. (4). One can then derive the fluid velocity $\mathbf{v}$ at the membrane surface by solving perturbatively Eq. (4). For $q \ll \kappa^{ \pm}$, and up to first order in the membrane undulation, we find that $v_{z}(q, z=0)=v_{z}^{+}(q, z=0)+v_{z}^{-}(q, z=0)$ with

$$
v_{z}^{ \pm}(q, z=0)=\frac{q h(q)}{\epsilon \eta}\left(-\frac{3}{8} q \frac{\sigma^{ \pm}}{\kappa^{ \pm}}+\frac{1}{2} q^{2} \frac{\sigma^{ \pm 2}}{\kappa^{ \pm 2}}-\frac{3}{32} q^{4} \frac{\sigma^{ \pm 2}}{\kappa^{ \pm 3}}\right) .
$$

This normal velocity could be derived from an effective free energy density

$$
F_{\text {eff }}(q)=\left[\Sigma q^{2}+\Gamma q^{3}+K q^{4}\right] h^{2}(q) / 2
$$

with $\Sigma=3\left(\sigma^{+^{2}} / \kappa^{+}+\sigma^{-2} / \kappa^{-}\right) / 2 \epsilon, K=3\left(\sigma^{+^{2}} / \kappa^{+3}+\sigma^{-2} / \kappa^{-3}\right) / 8 \epsilon$ and $\Gamma=-2\left(\sigma^{+^{2}} / \kappa^{+^{2}}+\right.$ $\left.\sigma^{-2} / \kappa^{-2}\right) / \epsilon$. Thus, if nonthermal noise can be neglected, the fluctuation spectrum is $\left\langle|h(q)|^{2}\right\rangle=$ 
$k_{B} T /\left(\left(\Sigma_{0}+\Sigma\right) q^{2}+\Gamma q^{3}+\left(K_{0}+K\right) q^{4}\right)$, where we have assumed that the membrane has, in addition to the electrostatic contribution, a bare tension $\Sigma_{0}$ and a bare bending modulus $K_{0}$.

Let us now discuss the physical significance of this result:

(i) The first important point is that this derivation of the fluctuation spectrum is independent of the origin of the ion fluxes. In particular, it applies also to the case where the ion fluxes are produced internally by active pumps, in the absence of any external potential difference $V=0$ and concentration gradients $n^{-}=n^{+}$. Alternatively, it also applies when the fluxes are produced by concentration gradients $n^{-} \neq n^{+}$in the absence of any external potential difference $V=0$, provided that the two ions are different (we must require $G_{1} \neq G_{2}$ or $D_{1} \neq D_{2}$ so that $\sigma^{ \pm} \neq 0$ ).

(ii) In addition to the renormalizations of the membrane tension and bending modulus, a term proportional to $q^{3}$ in the effective free energy is present. This term does not exist for an equilibrium membrane in an electrolyte solution, as the electrostatic potential only contains even powers of $q$. It is due to the hydrodynamic couplings, which induce a nonanalytic dependence on $q$. Furthermore, the negative sign of $\Gamma$ suggests the possibility of an instability of the membrane [14]. To see this, let us first consider a free membrane. There is no tension, and $\Sigma_{t}=\Sigma_{0}+\Sigma=0$. The membrane is unstable because the average squared undulation is negative at low wave vector. In the case of a vesicle, there is a finite tension, which can be seen as a Lagrange multiplier ensuring the conservation of its area. The vesicle is unstable if the total tension is low enough to satisfy the condition $\Sigma_{t}<\Gamma^{2} / 4\left(K_{0}+K\right)$. This instability occurs at a wave vector $q_{c}=-\Gamma / 2\left(K_{0}+K\right)$ suggesting a periodic deformation of the membrane at this characteristic wave vector. As expected, the instability threshold for the membrane tension increases with the ion fluxes or the applied potential difference.

(iii) The value of the renormalized membrane tension $\Sigma$ can also be obtained from the zeroth order electrostatic potential, with a simple mechanical argument. Indeed, the surface tension is related to the Maxwell stress by [15]:

$$
\Sigma=-\epsilon \int_{-L / 2}^{L / 2}\left(E_{z}^{(0)}\right)^{2}(z) d z+\frac{\epsilon L}{2}\left[\left(E_{z}^{(0)}\right)^{2}(z \rightarrow \infty)+\left(E_{z}^{(0)}\right)^{2}(z \rightarrow-\infty)\right]
$$

Note that in the r.h.s. of Eq 7 the second term cancels a contribution proportional to $L$ in the first term, which originates from the existence of a pressure gradient in the fluid.

(iv) For order-of-magnitude estimates, we obtain with the parameters reported in Fig 2 $\Sigma=3.2 \cdot 10^{-16} \mathrm{Jm}^{-2}, \Gamma=-10^{-24} \mathrm{Jm}^{-1}$ and $K=10^{-13} k_{B} T$. Although the ion flux is consequent and typical of ion channels, the moduli $\Sigma, \Gamma$ and $K$ are very small due to the strong dependance of these moduli on $\kappa^{-1}$, which is here only $2.3 \mathrm{~nm}$. As we show below, these low values also reflect the fact that sofar, we have neglected the bilayer character of the membrane and its finite capacitance.

Bilayer of finite thickness and finite dielectric constant. - In this section, we extend the calculation to a bilayer of finite thickness $d$ and dielectric constant $\epsilon_{m}<\epsilon$. There is then an electrical coupling between the membrane and the surrounding electrolyte, with a strength measured by the parameter $t=\epsilon_{m} /(\kappa d \epsilon)[3,16]$. For equilibrium membranes, the importance of this coupling is discussed in refs. $[17,18]$. For the sake of simplicity, we only discuss the case of symmetric electrolytes: $n^{-}=n^{+}, D_{1}=D_{2}=D$, and $G_{1}=G_{2}=G$ because we intend to provide the full solution and more details in a longer paper. We denote by $\phi_{m}$ the internal potential and $\phi$ the electrolyte potential. When $t \neq 0$, the boundary conditions at 
EUROPHYSICS LETTERS

the membrane are modified, they are now:

$$
\begin{gathered}
\epsilon \partial_{z} \phi^{(0)}(z \rightarrow \pm d / 2)=\epsilon_{m} \partial_{z} \phi_{m}^{(0)}(z \rightarrow \pm d / 2) \\
\phi^{(0)}(z \rightarrow \pm d / 2)=\phi_{m}^{(0)}(z \rightarrow \pm d / 2)
\end{gathered}
$$

where the first equation is the continuity condition for the normal electric displacement and the second equation is the continuity condition of the potential. With these assumptions, we find that the current-voltage relation given in Eq. 3 still holds for any value of $t$. Furthermore, the electric field for $z>d / 2$ (resp. $z<-d / 2)$ is $E_{z}^{(0)}(z)=-\sigma / \epsilon-\tilde{\sigma} / \epsilon \exp (\kappa(z+d / 2))$ (resp. $\left.E_{z}^{(0)}(z)=-\sigma / \epsilon-\tilde{\sigma} / \epsilon \exp (\kappa(-z+d / 2))\right)$, while the internal field is constant and equal to $E_{m}=-(\sigma+\tilde{\sigma}) /(t \kappa d \epsilon)$. We have introduced $\sigma$ the surface charge on the positive electrode, $\sigma$, which is related to the electrical current $i=i_{1}=i_{2}$ in a way similar to Eq. 2 by $\sigma=-2 i / \kappa^{2} D$, and $\tilde{\sigma}$ defined by $\tilde{\sigma}=\int_{0}^{\infty} \rho^{+}(z) d z$. These two surface charges are related by $\tilde{\sigma} / \sigma=\left(D \kappa^{3} \epsilon t-2 G\right) / 2(2 t+1) G$. Note that only the diffusion time of the ions within a Debye layer enters in $\sigma$ whereas $\tilde{\sigma}$ also contains the RC characteristic time of the membrane.

Using Eq. 7 and the equations above, the surface tension can now be written as the sum of an internal contribution $\Sigma_{i n}=-\epsilon_{m} d E_{m}^{2}=-(\sigma+\tilde{\sigma})^{2} / t \kappa \epsilon$ and an external contribution $\Sigma_{\text {out }}=\left(-\tilde{\sigma}^{2}-4 \sigma \tilde{\sigma}+\kappa d \sigma^{2}\right) / \epsilon \kappa$. The negative contribution $\Sigma_{\text {in }}$ is known as the Lippmann tension [19] and is usually larger in absolute value than $\Sigma_{\text {out }}$. Because of $\Sigma_{\text {in }}$, the total membrane tension $\Sigma_{0}+\Sigma_{\text {in }}+\Sigma_{\text {out }}$ can become negative at some critical value of the internal field $E_{m}$, which leads to the instabilities discussed in ref. [20]. As discussed in the previous section, the surface tension $\Sigma$ can also be recovered by solving Stokes equation at first order in the membrane height with the appropriate electrostatic forces as source terms. Care must be used here as the usual definition of the (bulk) electrostatic force $\rho \mathbf{E}$ holds only inside a medium with a homogeneous dielectric constant, but not at the boundaries between two dielectric media, where the jump in dielectric constant is associated with a localized force. These difficulties can be resolved by calculating the electrostatic force directly with the Maxwell stress tensor [21]. From the solution of Stokes equation, the velocity is obtained everywhere in the domain $|z|>d / 2$. By extrapolating this velocity at $z=0$, an effective free energy of the same form as in Eq. (6) is obtained, with the same tension $\Sigma$ as calculated from Eq. 7 . We also obtain the moduli $\Gamma=\sigma \tilde{\sigma}\left(8+\kappa^{2} d^{2}+4 \kappa d\right) / 2 \kappa^{2} \epsilon$ and $K$ which is a complicated quadratic function of $\sigma$ and $\tilde{\sigma}$.

We now discuss the limit of small conductance $G \rightarrow 0$ which is of experimental relevance. In this limit, $\sigma=0$ since there is no current in the medium $i=0$. The membrane is a capacitor of surface charge $\tilde{\sigma}=-\epsilon_{m} E_{m}=V \epsilon \kappa t /(1+2 t)$. This means that the equivalent circuit is made of three planar capacitors in series, one being the membrane (of capacitance per unit area $\epsilon_{m} / d$ ) and the other two corresponding to the Debye layers on each side (of capacitance $\epsilon \kappa$ per unit area), making up a total capacitance $C=\epsilon \kappa t /(2 t+1)$. Now $\Sigma_{\text {in }}=-\tilde{\sigma}^{2} / t \kappa \epsilon, \Sigma_{\text {out }}=-\tilde{\sigma}^{2} / \kappa \epsilon$, and $\Gamma=0$. In this limit, $\Gamma=0$ since the system is at equilibrium and therefore the effective free energy must be of the Helfrich form. With the values of the parameters used in Fig. 2 except for $G$ which we take to be $\mathrm{G}=0$ and $d=5 \mathrm{~nm}, \epsilon_{m} / \epsilon=1 / 40$, we obtain $t=3.3 \cdot 10^{-3}$ and $\kappa d=7.4, \Sigma_{\text {in }}=-8.4 \cdot 10^{-6} \mathrm{Jm}^{-2}, \Sigma_{\text {out }}=-1.0 \cdot 10^{-7} \mathrm{Jm}^{-2}, \Gamma=0$ and $K=0.011 k_{B} T$. If we use instead $G=10 \Omega^{-1} / \mathrm{m}^{2}$ a typical value for ion channels, the order of magnitude of the tension and bending modulus are unchanged and a small value of $\Gamma=8.2 \cdot 10^{-20} \mathrm{Jm}^{-1}$ is found. This indicates that the capacitor model with $G=0$ is a good approximation to calculate the moduli in this case. The importance of capacitive effects is confirmed by the fact that the values of the moduli obtained here are much larger than the ones obtained previously in the case of zero thickness. Furthermore, by varying the ionic strength in the case where ion transport is present with $G \neq 0$, we have found that the capacitor model holds at high ionic 
strength but become invalid at low ionic strength where ion transport has a stronger impact on the moduli. This will be discussed in a future work.

Conclusions. - In conclusion, we have analyzed the steady-state fluctuations of a membrane driven out-of-equilibrium by an applied DC electric field or by concentration gradients. One of our most notable results is the presence of a new term proportional to $q^{3}$ in the fluctuation spectrum that we interpret as arising from hydrodynamics couplings. For a free membrane or for a vesicle of zero thickness and negligible dielectric constant, we have found a negative value for $\Gamma$ which gives rise to a finite wavelength instability. For a bilayer, we recover a known zero wavevector instability, when the surface tension becomes negative. Since $\Gamma$ is positive in this case, the other instability at finite wavelength is absent for a bilayer. We have also analyzed the role of capacitive effects, and found that they lead to a dominant contribution in the electrostatic part of the surface tension and bending modulus at high ionic strength. The analysis presented here suggests directions for future study. In particular, it would be interesting to go beyond the linear approximation for the electrostatic potential and the ion concentrations, and discuss with more details the implications for the dynamics of active membranes containing for instance gated or mechano-sensitive ion channels.

We thank D. Andelman for pointing out to us ref. [4], P. Sens, H. Aranda-Espinoza, J.B. Fournier, A. Ajdari, and J. Prost for many useful discussions. M. C. L. acknowledges financial support of a Human Frontier Science Foundation grant.

\section{REFERENCES}

[1] E. Edidin, Nat. Rev. Mol. Cell. Biol.4, 414 (2003).

[2] P. Pincus, J. F. Joanny, D. Andelman, Europhysics letters 11, 763 (1990).

[3] M. Winterhalter and W. Helfrich, J. Phys. Chem., 92, 6865 (1988).

[4] R. E. Goldstein, A. I. Pesci, V. Romero-Rochin, Phys. Rev. A 41, 5504 (1990).

[5] P. Girard, F. Jülicher, J. Prost, Eur. Phys. J. E Soft Matter 14, 387 (2004).

[6] J.-B. Manneville, P. Bassereau, S. Ramaswamy, and J. Prost, Phys. Rev. E 64, 021908 (2001).

[7] M. Leonetti, E. Dubois-Violette, and F. Homblé, PNAS 101, 10243 (2004).

[8] D. Lacoste and A. W. C. Lau, Europhys. Lett. , 70 (3), 418 (2005).

[9] M. A. Lomholt, Phys. Rev. E, 73, 061913 (2006).

[10] A. Ajdari, Phys. Rev. Lett., 75, 755 (1995).

[11] B. Hille "Ion Channels of Excitable Membranes", Sinauer Press, Sunderland MA (2001).

[12] E. Kandel, J. Schwartz and T. Jessel, "Principles of neural science", Mac Graw Hill, Ney York (2001).

[13] For an extensive review, see U. Seifert, Adv. Phys. 46, 13 (1997).

[14] S. T. Milner, J.-F. Joanny and P. Pincus, Europhys. Lett., 9, 495 (1989).

[15] J. S. Rowlinson and B. Widom, Molecular Theory of Capillarity (Oxford, 1982).

[16] D. Andelman, Electrostatic properties of membranes: the Poisson-Boltzmann Theory in Handbook of Biological Physics edited by R. Lipowsky and E. Sackmann (1995).

[17] M. Kiometzis and H. Kleinert, Phys. Lett. A, 140, 520 (1989).

[18] T. Chou, M. V. Jaric and E. Siggia, Biophysical Journal, 72, 2042 (1997).

[19] P-C. Zhang, A. M. Keleshian, and F. Sachs, 413, Nature, 428 (2001).

[20] P. Sens and H. Isambert, Phys. Rev. Lett., 88, 128102 (2002).

[21] L. D. Landau, E. M. Lifshitz and L. P. Pitaevskii, Electrodynamics of continuous media (Elsevier, New York, 2002). 